\title{
Viscoelastic Phase Separation in Shear Flow
}

\author{
Tatsuhiro Imaeda ${ }^{1}$, Akira Furukawa ${ }^{2}$, and Akira Onuki ${ }^{2}$ \\ ${ }^{1}$ Aichi Gakusen University, Toyota 471-8532, Japan \\ ${ }^{2}$ Department of Physics, Kyoto University, Kyoto 606-8502, Japan
}

(Dated: October 29, 2018)

\begin{abstract}
We numerically investigate viscoelastic phase separation in polymer solutions under shear using a time-dependent Ginzburg-Landau model. The gross variables in our model are the polymer volume fraction and a conformation tensor. The latter represents chain deformations and relaxes slowly on the rheological time giving rise to a large viscoelastic stress. The polymer and the solvent obey two-fluid dynamics in which the viscoelastic stress acts asymmetrically on the polymer and, as a result, the stress and the diffusion are dynamically coupled. Below the coexistence curve, interfaces appear with increasing the quench depth and the solvent regions act as a lubricant. In these cases the composition heterogeneity causes more enhanced viscoelastic heterogeneity and the macroscopic stress is decreased at fixed applied shear rate. We find steady two-phase states composed of the polymer-rich and solvent-rich regions, where the characteristic domain size is inversely proportional to the average shear stress for various shear rates. The deviatoric stress components exhibit large temporal fluctuations. The normal stress difference can take negative values transiently at weak shear.

PACS numbers: PACS numbers: $64.75 .+\mathrm{g}$, 83.60.Df, 83.60.Rs, 83.80.Rs
\end{abstract}

\section{INTRODUCTION}

In phase-separating polymer systems, domain morphologies are influenced by a number of factors, including the molecular weights, the composition, closeness to the critical point, and viscoelasticity [1, 2]. Particularly when the two components have distinctly different viscoelastic properties as in semidilute polymer solutions and polymer blends of long and short chains, unique interplays emerge between viscoelasticity and thermodynamic instability. In such asymmetric systems, salient effects observed in experiments are as follows. First, in early stage spinodal decomposition, the kinetic coefficient $L(q)$ depends on the wave number $q$ as $L(q) / L(0) \sim\left(\xi_{\mathrm{ve}} q\right)^{-2}$ for $q$ larger than the inverse of a viscoelastic length $\xi_{\mathrm{ve}}[3,4]$, where $\xi_{\text {ve }}$ can be much longer than the gyration radius 5 . Second, in late stage spinodal decomposition, sponge-like network structures appear transiently in the asymmetric case [6, 7]. The physical origin of these effects is now ascribed to the stress-diffusion coupling in viscoelastic binary mixtures [5]. This coupling is predicted to give rise to various effects including non-exponential decay in dynamic light scattering [2, 5] and shear-induced fluctuation enhancement. In the case of spinodal decomposition, the consequences of the dynamic coupling were studied analytically in the linear growth regime 8 and numerically in the late stage coarsening [9, 10, 11].

Flow effects on phase separation in polymeric systems are even more dramatic [1, 12, 13]. In this paper we consider a simple shear flow with mean velocity profile

$$
\langle\boldsymbol{v}\rangle=\dot{\gamma} y \boldsymbol{e}_{x},
$$

where the flow is in the $x$ direction, $\boldsymbol{e}_{x}$ being the unit vector along the $x$ axis, and the mean velocity gradient $\dot{\gamma}$ is in the $y$ direction. Application of shear or extensional flow to viscoelastic systems in one-phase states sometimes induces a strong increase of the turbidity, indicating shear-induced composition heterogeneities or demixing. This is in marked contrast to shear-induced homogenization or mixing observed in near-critical fluids [14, 15] and in ternary polymer mixtures [16, 17]. In systems exhibiting shear-induced mixing, the entanglement effects are not severe and the hydrodynamic interaction is suppressed by shear [2, 13]. In semidilute polymer solutions near the coexistence curve with high molecular weights $\left(M \gtrsim^{2 \times 10^{6}}\right.$ ), recent scattering experiments have most unambiguously detected shear-induced demixing [18, 19, 20, 21, 22, 23, 24, 25]. Rheological effects in sheared polymer solutions are also conspicuous, which include large stress fluctuations upon demixing by shear [26, 27] and a second overshoot in the shear stress as a function of time after application of shear [20, 28]. Here microscope pictures of composition heterogeneities in polymer solutions and asymmetric polymer blends under shear are informative [29, 30].

Theoretically, for sheared polymer solutions, relevance of the dynamical coupling was first pointed out by Helfand and Fredrickson [31]. Some linear calculations for small fluctuations were also performed in GinzburgLandau schemes, where a conformation tensor represents the chain deformations 32, 33, 34, 35. Numerical analysis (still in two dimensions) using such schemes gave insights into the nonlinear shear effects [13, 36, 37, 38, 39]. That is, slightly above the coexistence curve, composition heterogeneities $\delta \phi$ on mesoscopic spatial scales emerge with increasing $\dot{\gamma}$. The amplitude of $\delta \phi$ can even be of the order of the average $\langle\phi\rangle$, while there are no clear interfaces. If this takes place, the system becomes turbid resulting in shear-induced phase separation or demixing observed above the coexistence curve. We remark that similar Ginzburg-Landau models [40, 41] have been used to analyze shear-banding effects in wormlike micellar systems [42, 43, 44]. 
In entangled polymers the rheological relaxation time $\tau$ can be very long [45], so experiments in the Newtonian and non-Newtonian regimes are both possible, where the Deborach number $D e=\dot{\gamma} \tau$ is larger or smaller than 1, respectively. In semidilute solutions, the strong composition-dependence of the solution viscosity $\eta=\eta(\phi)$ can lead to shear-induced fluctuation enhancement for weak shear $\dot{\gamma} \tau \ll 1$ [31], while the normal stress effect comes into play in the non-Newtonian regime $\dot{\gamma} \tau \geq 1$ [2, 21, 32].

Without viscoelasticity a number of groups have performed simulations of phase separation in sheared simple fluids [46, 47]. However, simulations have been still rare for sheared viscoelastic fluids [13, 36, 37, 38, 39]. In this paper, we will examine nonlinear dynamic regimes of sheared polymer solutions in theta solvent below the coexistence curve. Both in simple and polymeric fluids, if the system is quenched below the coexistence curve under shear, the domain growth is eventually stopped and dynamically steady states are realized. For Newtonian fluids dynamics in sheared two-phase states have long been studied [2, 17, 48, 49], but for viscoelastic fluids such nonequilibrium effects remain almost unexplored.

The organization of this paper is as follows. In Sec. II, we will explain our theoretical scheme. In Sec. III, we will present our numerical results in two dimensions below the coexistence curve for various shear rates and polymer volume fractions. Section IV summarizes new results and gives some predictions.

\section{THEORETICAL BACKGROUND}

In this section we briefly survey our theoretical framework to discuss viscoelastic phase separation under the shear flow (1.1). The gross variables in our model are the polymer volume fraction $\phi$ and a conformation tensor $\overleftrightarrow{W}$. The latter represents chain deformations and relaxes slowly on the rheological time giving rise to a large viscoelastic stress. In Sec. II A we first present the viscoelastic Gintzburg-Landau free energy, and then in Sec. II B the dynamic model is given. The rheological properties of our dynamic equations are also discussed in Sec. II C. See Refs. [2, 13] for the details of our theoretical framework.

\section{A. Viscoelastic Ginzburg-Landau Free Energy}

Phase behavior of polymer solutions near the coexistence curve is usually described in term of the FloryHuggins free energy density for the polymer volume fraction $\phi$ assumed to be much smaller than 1 [45],

$$
f_{\mathrm{FH}}=\frac{k_{\mathrm{B}} T}{v_{0}}\left[\frac{\phi}{N} \ln \phi+\left(\frac{1}{2}-\chi\right) \phi^{2}+\frac{1}{6} \phi^{3}\right],
$$

where $v_{0}$ is the volume of a monomer $\left(=a^{3}\right.$ with $a$ being the monomer size in three dimensions) and $\chi$ is the interaction parameter dependent on the temperature $T$ (being equal to $1 / 2$ at the theta condition). At the critical point we have $\phi=\phi_{\mathrm{c}}=N^{-1 / 2}$ and $N^{1 / 2}(1-2 \chi)=-2$. In the following it is convenient to scale $\phi$ and $2 \chi-1$ as

$$
\Phi=\phi / \phi_{\mathrm{c}}, \quad u=N^{1 / 2}(2 \chi-1) .
$$

In Fig. 1 we show the phase diagram in the plane of $\phi / \phi_{\mathrm{c}}$ and $N^{1 / 2}(1-2 \chi)=-u$. The spinodal curve in Fig.1 is obtained from $\left(\partial^{2} f_{\mathrm{FH}} / \partial \phi^{2}\right)_{T}=0$ and is written as $u=\Phi+\Phi^{-1}$.

To describe the viscoelastic effects on the composition inhomogeneities, it is convenient to introduce a tensor dynamic variable $\stackrel{\leftrightarrow}{W}=\left\{W_{i j}\right\}$, which is a symmetric tensor representing chain conformations undergoing deformations [32, 33, 34, 50, 51]. As shown in the following, the deviation $\delta W_{i j}=W_{i j}-\delta_{i j}$ gives rises to a network stress.

The Ginzburg-Landau free energy functional due to the fluctuations of $\phi$ and $\overleftrightarrow{W}$ is written as

$$
F=\int d \boldsymbol{r}\left[f_{\mathrm{FH}}+\frac{1}{2} C|\nabla \phi|^{2}+\frac{1}{4} G Q(\stackrel{\leftrightarrow}{W})\right]
$$

The coefficient $C$ of the gradient term is calculated in the random phase approximation [45] in the semidilute regime as

$$
C=\left(k_{\mathrm{B}} T / 18 v_{0}\right) a^{2} / \phi .
$$

We shall see that $G$ has the meaning of the shear modulus for small deformations changing rapidly compared with the rheological relaxation time $\tau$. It is assumed to be of the scaling form,

$$
G=\left(k_{\mathrm{B}} T / v_{0}\right) g \phi^{\alpha},
$$

where $g$ is an important dimensionless parameter in our simulations, and is order of 1 in theta solvent 2, 13. Although experiments indicated $\alpha \cong 2.25$ [52, 53], we will set $\alpha=3$ for simplicity. The simplest form for $Q(\stackrel{\leftrightarrow}{W})$ is given by

$$
Q(\stackrel{\leftrightarrow}{W})=\sum_{i j}\left(\delta W_{i j}\right)^{2}
$$

\section{B. Dynamic Equations}

We next present the dynamic equations assuming the two-fluid dynamics for the polymer and the solvent with the velocities, $\boldsymbol{v}_{\mathrm{p}}$ and $\boldsymbol{v}_{\mathrm{s}}$, respectively, including the new variable $\overleftrightarrow{W}$ [32, 33, 34, 40]. These equations may be treated as Langevin equations with thermal noise terms, but we will neglect the noise terms hereafter. Formal frameworks for viscoelastic fluids have also been discussed in the literature [50, 51]. 
We assume that the mass densities of pure polymer and solvent are the same. Then the polymer mass fraction $\rho_{\mathrm{p}} / \rho$ coincides with $\phi$. It obeys

$$
\begin{aligned}
\frac{\partial}{\partial t} \phi & =-\nabla \cdot\left(\phi \boldsymbol{v}_{\mathrm{p}}\right) \\
& =-\nabla \cdot(\phi \boldsymbol{v})-\nabla \cdot[\phi(1-\phi) \boldsymbol{w}]
\end{aligned}
$$

where

$$
\boldsymbol{w}=\boldsymbol{v}_{\mathrm{p}}-\boldsymbol{v}_{\mathrm{s}}
$$

is the relative velocity between polymer and solvent. The mean velocity $\boldsymbol{v}$ is defined by

$$
\boldsymbol{v}=\phi \boldsymbol{v}_{\mathrm{p}}+(1-\phi) \boldsymbol{v}_{\mathrm{s}}
$$

The two velocities $\boldsymbol{v}_{\mathrm{p}}$ and $\boldsymbol{v}_{\mathrm{S}}$ are expressed as $\boldsymbol{v}_{\mathrm{p}}=\boldsymbol{v}+$ $(1-\phi) \boldsymbol{w}$ and $\boldsymbol{v}_{\mathrm{s}}=\boldsymbol{v}-\phi \boldsymbol{w}$. For simplicity, we assume the incompressibility condition for the average velocity,

$$
\nabla \cdot \boldsymbol{v}=0
$$

On the other hand, $\nabla \cdot \boldsymbol{w}$ is nonvanishing in general and gives rise to diffusion in (2.7) for small deviations around equilibrium.

Because $\stackrel{\leftrightarrow}{W}$ represents the network deformation, its motion is determined by the polymer velocity $\boldsymbol{v}_{\mathrm{p}}$ and its simplest dynamic equation is of the form,

$$
\begin{aligned}
{\left[\frac{\partial}{\partial t}\right.} & \left.+\boldsymbol{v}_{\mathrm{p}} \cdot \nabla\right] W_{i j}-\sum_{k}\left(D_{i k} W_{k j}+W_{i k} D_{j k}\right) \\
& =-\frac{1}{\tau^{*}} \delta W_{i j}
\end{aligned}
$$

where $D_{i j}=\partial v_{\mathrm{p} i} / \partial x_{j}$ is the gradient of the polymer velocity. The left hand side of (2.11) is called the upper convective time derivative in the rheological literature [54], which keeps the frame invariance of the tensor properties of $W_{i j}$. In (2.21) below, we will assume that the relaxation time $\tau^{*}$ on the right hand side depends on $\delta W_{i j}$ as well as $\phi$. The usual rheological relaxation time $\tau$ is obtained in the Newtonian limit:

$$
\tau=\lim _{\delta \overleftrightarrow{W} \rightarrow 0} \tau^{*}
$$

In the problem of shear-banding flow, some authors replaced $1 / \tau^{*}$ in (2.11) by $\left(1 / \tau^{*}\right)\left(1-\ell^{2} \nabla^{2}\right)$ [4, 44].

The total stress tensor $\stackrel{\leftrightarrow}{\Pi}=\left\{\Pi_{i j}\right\}$ is expressed as [2, 13]

$$
\begin{aligned}
\Pi_{i j} & =p \delta_{i j}+C\left(\nabla_{i} \phi\right)\left(\nabla_{j} \phi\right)-\sigma_{\mathrm{p} i j} \\
& -\eta_{0}\left(\nabla_{i} v_{j}+\nabla_{j} v_{i}\right),
\end{aligned}
$$

where $p$ is a pressure, $\nabla_{i}=\partial / \partial x_{i}, \stackrel{\leftrightarrow}{\sigma}_{\mathrm{p}}=\left\{\sigma_{\mathrm{p} i j}\right\}$ is the network stress arising from the deviation $\delta W_{i j}$, and the last term is the viscous stress tensor with $\eta_{0}$ being the solvent viscosity. Assuming low Reynolds number flows and setting $\partial \boldsymbol{v} / \partial t=\mathbf{0}$ (the Stokes approximation), we obtain 13 ]

$$
\nabla \cdot \overleftrightarrow{\Pi}=-\nabla p_{1}+\mathcal{F}_{\mathrm{p}}+\eta_{0} \nabla^{2} \boldsymbol{v}=\mathbf{0}
$$

where $p_{1}=p-\phi \delta F / \delta \phi+f_{\mathrm{FH}}+C|\nabla \phi|^{2} / 2$ ensures the incompressibility condition (2.10). The $\mathcal{F}_{\mathrm{p}}$ is the force density acting on the polymer due to the fluctuation of $\phi$ and $\overleftrightarrow{W}$ of the form,

$$
\mathcal{F}_{\mathrm{p}}=-\phi \nabla \frac{\delta F}{\delta \phi}-\frac{1}{4} Q \nabla G+\nabla \cdot \stackrel{\leftrightarrow}{\sigma}_{\mathrm{p}}
$$

The network stress tensor in (2.13) is expressed as

$$
\sigma_{\mathrm{p} i j}=G \sum_{k} W_{i k} \delta W_{k j}+\frac{1}{4} G Q \delta_{i j}
$$

To express $\boldsymbol{v}_{\mathrm{p}}$ and $\boldsymbol{v}_{\mathrm{s}}$ in terms of $\phi$ and $\overleftrightarrow{W}$, we assume slow processes and neglect the acceleration or inertial terms in the two-fluid dynamic equations [5]. By setting $\partial \boldsymbol{w} / \partial t=\mathbf{0}$, we obtain

$$
\boldsymbol{w}=\frac{1-\phi}{\zeta} \mathcal{F}_{\mathrm{p}}
$$

where $\zeta$ is the friction coefficient between polymer and solvent and is estimated as $\zeta \sim 6 \pi \eta_{0} \xi_{b}^{-2} \sim \eta_{0} \phi^{2} / a^{2}$ in the semidilute solution in terms of the blob size $\xi_{b}$. The mean velocity $\boldsymbol{v}$ is expressed as

$$
\boldsymbol{v}=\langle\boldsymbol{v}\rangle+\left[\frac{1}{-\eta_{0} \nabla^{2}} \mathcal{F}_{\mathrm{p}}\right]_{\perp},
$$

where $\langle\boldsymbol{v}\rangle$ is the mean flow such as the shear flow in (1.1), $[\cdots]_{\perp}$ denotes taking the transverse part (whose Fourier component is perpendicular to the wave vector) and the inverse operation $\left(-\eta_{0} \nabla^{2}\right)^{-1}$ may be expressed in terms of the Oseen tensor in the limit of large system size [2].

In the following, we make our eqations dimensionless by measuring space and time in units of $\ell$ and $\tau_{0}$ defined by

$$
\ell=\frac{a N^{1 / 2}}{2 \sqrt{18}}, \quad \frac{1}{\tau_{0}}=\frac{4 k_{\mathrm{B}} T}{\eta_{0} v_{0} N^{3 / 2}},
$$

where $\ell$ is of the order of the gyration radius, and the time $\tau_{0}$ is the conformation relaxation time of a single chain in the dilute case. In our simulations the mesh size in numerical integration will be set equal to $\ell$. The velocities will be measured in units of $\ell / \tau_{0}$ and the stress components given in (2.13) will be measured in units of

$$
\sigma_{0}=k_{\mathrm{B}} T /\left(v_{0} N^{3 / 2}\right)=\eta_{0} /\left(4 \tau_{0}\right) .
$$

To avoid cumbersome notation, in the follwoing, we use the same symbols for $t, \boldsymbol{r}, \nabla$, and the velocities even after rescaling. 


\section{Rheological quantities}

The conformation tensor $\stackrel{\leftrightarrow}{W}$ obeys (2.11) with $\tau^{*}$ being replaced by $\tau^{*} / \tau_{0}$ in the dimensionless form. Following Ref. 13] we assume

$$
\tau^{*} / \tau_{0}=\left(\Phi^{4}+0.2\right) /(1+Q)
$$

where $Q=Q(\stackrel{\leftrightarrow}{W})$ is defined by (2.6) and the factor $1 /(1+Q)$ accounts for quickening of the stress relaxation under large deformations [55]. Similarly, some authors assumed a deformation-dependent stress relaxation time in the rheological constitutive equations [54, 56].

Consequences of (2.21) are as follows. (i) In the dilute regime we have $\tau^{*} \cong 0.2 \tau_{0}$. (ii) The relaxation time $\tau$ in (2.12) in the linear response regime becomes

$$
\tau=\tau_{0}\left(\Phi^{4}+0.2\right)
$$

(iii) The zero-frequency linear viscosity becomes

$$
\eta / \eta_{0}=1+\frac{1}{4} g \Phi^{3} \tau / \tau_{0} \cong 1+\frac{1}{4} g \Phi^{7},
$$

where the small number 0.2 in $\tau$ is omitted. (iv) Let us consider a homogeneous state under shear, where $\boldsymbol{v}_{\mathrm{p}}=$ $\boldsymbol{v}=\dot{\gamma} y \boldsymbol{e}_{x}$ and $\phi=$ const. In the high shear limit $\dot{\gamma} \tau \gg 1$, by solving (2.11) we obtain shear thinning behavior,

$$
\sigma_{\mathrm{p} x y} \sim g \Phi^{3}(\dot{\gamma} \tau)^{3 / 5}, \quad N_{\mathrm{p} 1} \sim g \Phi^{3}(\dot{\gamma} \tau)^{4 / 5} .
$$

Non-Newtonian behavior can arise from the factor $1 /(1+$ $Q)$ in $\tau^{*}$ even in homogeneous states.

Next we give dimensionless forms of the stress components for general inhomogeneous cases. From (2.14) and (2.16) the shear stress $\sigma_{x y}$ and the normal stress difference $N_{1}=\sigma_{x x}-\sigma_{y y}$ in units of $\sigma_{0}$ are written as

$$
\begin{aligned}
\sigma_{x y}= & \sigma_{\mathrm{p} x y}-\frac{4}{\Phi} \nabla_{x} \Phi \nabla_{y} \Phi+4\left(\nabla_{x} v_{y}+\nabla_{y} v_{x}\right), \\
N_{1}= & N_{\mathrm{p} 1}-\frac{4}{\Phi}\left(\nabla_{x} \Phi \nabla_{x} \Phi-\nabla_{y} \Phi \nabla_{y} \Phi\right) \\
& +8\left(\nabla_{x} v_{x}-\nabla_{y} v_{y}\right),
\end{aligned}
$$

where $\sigma_{\mathrm{p} x y}$ and $N_{\mathrm{p} 1}$ are the network contributions,

$$
\begin{aligned}
\sigma_{\mathrm{p} x y} & =g \Phi^{3}\left(W_{x x}+W_{y y}-1\right) W_{x y} \\
N_{\mathrm{p} 1} & =g \Phi^{3}\left(W_{x x}+W_{y y}-1\right)\left(W_{x x}-W_{y y}\right)
\end{aligned}
$$

The second terms in (2.25) and 2.26) arise from inhomogeneity in $\Phi$ and give rise to the surface tension contributions in two-phase states [57]. The last terms are the usual viscous contributions.

The physical meaning of $\sigma_{\mathrm{p} x y}$ and $N_{\mathrm{p} 1}$ can be seen if they are related to the degree of chain extension. To this end let us decompose $\delta \overleftrightarrow{W}$ as

$$
\delta \overleftrightarrow{W}=w_{1} \boldsymbol{e}_{1} \boldsymbol{e}_{1}+w_{2} \boldsymbol{e}_{2} \boldsymbol{e}_{2}
$$

where $w_{1}$ and $w_{2}$ are the eigenvalues of $\delta \stackrel{\leftrightarrow}{W}$ with $w_{1} \geqq w_{2}$, and

$$
\boldsymbol{e}_{1}=(\cos \theta, \sin \theta), \quad \boldsymbol{e}_{2}=(-\sin \theta, \cos \theta)
$$

are the corresponding eigenvectors with $\theta$ being the angle between the stretched direction and the $x$ axis. We may assume $-\pi / 2<\theta \leqq \pi / 2$ without loss of generality. In terms of these quantities we obtain

$$
\begin{aligned}
\sigma_{\mathrm{p} x y} & =\frac{g}{2} \Phi^{3}\left(1+w_{1}+w_{2}\right)\left(w_{1}-w_{2}\right) \sin 2 \theta \\
N_{\mathrm{p} 1} & =g \Phi^{3}\left(1+w_{1}+w_{2}\right)\left(w_{1}-w_{2}\right) \cos 2 \theta .
\end{aligned}
$$

In weak shear $\tau \dot{\gamma} \ll 1$, we have

$$
w_{1}-w_{2} \sim \tau \dot{\gamma}, \quad \theta-\pi / 4 \sim \tau \dot{\gamma}
$$

so $\sin 2 \theta \cong 1$ and $\cos 2 \theta \sim \tau \dot{\gamma}$, leading to the well-known results $\sigma_{\mathrm{D} x y} \sim \eta \dot{\gamma}$ and $N_{1} \sim \eta \tau \dot{\gamma}^{2}$ in the Newtonian regime [54]. Here, for the analysis in Sec. III, we introduce an extension vector defined by

$$
\boldsymbol{w}(\boldsymbol{r}, t)=\left(w_{1}-w_{2}\right) \boldsymbol{e}_{1},
$$

whose magnitude and direction represent the degree of chain extension and the extended direction. In our simulations we shall see that the magnitudes of $w_{1}$ and $w_{2}$ are both considerably smaller than 1 at most space points. That is, the degree of extension is rather weak, but the network stress can overwhelm the viscous stress $\left(\propto \eta_{0}\right)$ because of the large factor $g \Phi^{3}$. This justifies the Gaussian form of $Q(\overleftrightarrow{W})$ in (2.6) in this work.

\section{NUMERICAL RESULTS}

We need numerical approach to understand the nonlinear regime of shear-induced phase separation. To this end we integrate our model equations, (2.7) and (2.11), in the previous section on a $256 \times 256$ lattice in two dimensions. The mesh size $\Delta x$ is set equal to $\ell$ in (2.19) [58]. We use a numerical scheme developed by one of the present authors 47, 59], which uses the deformed coordinates $x^{\prime}=x-\dot{\gamma} t y$ and $y^{\prime}=y$ and enables the FFT (fast Fourier transform) method to be carried out for shear flow. Here we impose the periodic boundary condition $f\left(x^{\prime}, y^{\prime}+L\right)=f\left(x^{\prime}+L, y^{\prime}\right)=f\left(x^{\prime}, y^{\prime}\right)$ for any quantity $f\left(x^{\prime}, y^{\prime}\right)$ in terms of the deformed coordinates.

As the initial condition at $t=0$ we assign Gaussian random numbers with variance (rms amplitude) 0.1 to $\Phi$ at the lattice points. Then the initial $\Phi$ at each point consists of the average $\langle\Phi\rangle$ and a random number. For $t>0$ we will solve the dynamic equations in the presence of shear without the random noise terms. Here, if quenching is is not very far above the coexistence curve, the initial variance is not very small and our choice can be appropriate [60]. 


\section{A. Crossover from Newtonian to viscoelastic fluids below the coexistence curve}

Even below the coexistence curve, if the shear rate is sufficiently strong $(\dot{\gamma} \tau \gg 1)$, the composition fluctuations vary in space gradually and there is no distinct phase separation. However, with increasing the quench depth (or $u$ ) and/or decreasing the shear rate $\dot{\gamma}$, the shear-induced composition fluctuations become composed of polymerrich and solvent-rich regions with sharp interfaces. Here we show that the domain morphology strongly depends on the shear modulus $G$.

In Fig. 2 we show phase separation patterns at $t=600$ and 2000 for $g=0$ (a), 0.01 (b), 0.1 (c), and 1 (d) after quenching at $t=0$, where $g$ represents the magnitude of $G$ in (2.5). The other parameters take common values as $\langle\Phi\rangle=2, u=3$, and $\dot{\gamma}=0.025$. The initial Deborach number before phase separation is given by $\dot{\gamma} \tau=\dot{\gamma} \Phi^{4}=$ 0.4. In the phase separated semidilute regions we have

$$
\Phi_{\mathrm{cx}} \cong 3.70
$$

for $u=3$ on the polymer-rich branch of the coexistence curve in Fig.1, so within the polymer-rich domains the viscosity ratio in (2.23) is given by 1 (a), 24.8 (b), 239 (c), and $2384(\mathrm{~d})$. We can see gradual crossover from the patterns of the Newtonian fluids to those of highly asymmetric viscoelastic fluids.

In Fig.3 we show time evolution of the inverse of the perimeter length density for $g=0,0.01,0.1$, and 1 , using the same conditions as in Fig.2. It may be regarded as the typical domain size $R(t)$. The domains continue to grow for $g=0$ and 0.01 within our simulation time $t<10^{4}$, but the growth is gradually slowed down with increasing $g$. For $g=1$ and 0.1 , we can see that the coarsening is nearly stopped.

\section{B. Domain size in two-phase flow}

Hereafter we fix $g$ at 1 . In Fig.4 we show time evolution of the domain size $R(t)$ for $\langle\Phi\rangle=2$ and $u=3$ at various shear rates $\dot{\gamma}=0.0005,0.005,0.025$, and 0.05 . Remarkably, the coarsening is faster for $\dot{\gamma}=0.005$ than for $\dot{\gamma}=0.0005$. This should be due to shear-induced coagulation of domains observed in near-critical fluids [13, 61], where shear accelerates collision and fusion of the domains. For shear rates larger than 0.025, dynamical steady states are realized, where there should be a balance between the thermodynamic instability and shearinduced domain breakup as in the Newtonian case [2, 13].

The characteristic domain size $R_{\mathrm{D}}$ in the steady states are of interest. In Newtonian immiscible mixtures under shear we have $R_{\mathrm{D}} \sim C_{\mathrm{New}} /\left\langle\sigma_{x y}\right\rangle$ in the low-Reynoldsnumber limit [2, 13, 16], where $C_{\text {New }}$ is of the order of the surface tension $\gamma[62]$. This formula follows from a balance between the surface energy density $\gamma / R_{\mathrm{D}}$ and the shear stress. Also in our non-Newtonian case Fig.5 suggests the same form,

$$
R_{\mathrm{D}} \sim C_{\mathrm{vis}} /\left\langle\sigma_{x y}\right\rangle
$$

at various $\dot{\gamma}$. The coefficient $C_{\text {vis }}$ is independent of $\dot{\gamma}$ but dependent on $\langle\Phi\rangle$ as 20,13 , and 11 for $\langle\Phi\rangle=2.5,2$, and 1.7, respectively. Fig.5 displays the product $\left\langle\sigma_{x y}\right\rangle(t) R(t)$ as a function of time after quenching at $g=1$ and $u=$ 3 with $\langle\Phi\rangle=2$ and $\langle\Phi\rangle=2.5$. The curves tend to composition-dependent constants independently of $\dot{\gamma}$ at long times to confirm (3.2). However, we cannot derive (3.2) using simple arguments in this paper.

In the viscoelastic case, as in (2.25) and (2.26), the stress consists of the three contributions. As shown in Fig.6 at $\dot{\gamma}=0.05$, the network stress $\sigma_{\mathrm{p} x y}$ dominates over the gradient contribution in the polymer-rich regions, whereas the network, gradient, and viscous ones are of the same order in the interface regions. The particularly large size of $\sigma_{\mathrm{p} x y}$ in the polymer-rich regions suggests that these regions should behave like percolated gels and mostly support the applied stress. On the other hand, the viscous contribution is very small in the polymerrich regions, but is nearly the sole contribution in the solvent-rich regions.

\section{Fine domains at strong shear}

For the largest shear rate $\dot{\gamma}=0.05$ in Fig.4, Fig.7 displays time evolution of the domains, where the polymerrich domains are percolated and the angle of extension $\theta$ defined by (2.29) and (2.30) is close to $\pi / 4$. These closely resemble the observed microscope pictures (in the $x z$ plane) 29, 30]. The profile of $\Phi$ at the bottom of Fig.7 shows that $\Phi$ becomes close to 0 in the solvent-rich regions, while it is around $\Phi_{\mathrm{ex}}$ in (3.1) in the polymer-rich regions.

Fig.8 displays the structure factor $S\left(k_{x}, k_{y}\right)$ of the composition fluctuations in the steady state in Fig.7. It has sharp double peaks along the $k_{x}$ axis with peak wave number $k_{\mathrm{p}} \cong(2 \pi / 256) \times 9$, although we cannot see marked anisotropy in the shapes of domains. The origin of the peaks is that the domains are connected and hence are aligned perpendicularly to the flow direction on the spatial scale of $2 \pi / k_{\mathrm{p}} \cong 28$.

In addition, we notice that the profile of $\Phi$ (bottom one in Fig.7) exhibit spike-like behavior around some extrema, where $\nabla \Phi$ varies over distances of order 5 [58]. These steep changes arise from convection due to the velocity fluctuations on such small scales in the nonlinear regime.

Fig.9 shows the average stress components $\left\langle\sigma_{x y}\right\rangle(t)$ and $\left\langle N_{1}\right\rangle(t)$ vs time, which exhibit pronounced overshoots and subsequent noisy behavior. Here the network contributions in (2.31) and (2.32) are much larger than the surface tension contributions arising from the second terms in (2.29) and (2.30) by at least one order of magnitude. This is consistent with Fig.6. Also shown is the average variance $\sqrt{\left\langle\delta \Phi^{2}\right\rangle}(t)$, which slowly increases over a long 
transient time of order 2000. This arises from desorption of solvent from the polymer-rich regions into the solventregions, as was observed by Tanaka and coworkers [6, 7].

\section{Large fluctuations at weak shear}

For the smaller shear rate $\dot{\gamma}=0.005$, Fig.10 demonstrates that the domain growth continues up to the system size in the simulation time $t=10^{4}$. Furthermore, comparing the two snapshots at $t=300$ and 500 , we can see that the domains are rotated as a whole in the early stage. Fig.11 shows that the chaotic temporal fluctuations of the stress are much more exaggerated than in Fig.9. Unusually the normal stress frequently takes negative values, while it is always positive at $\dot{\gamma}=0.05$ as shown in Fig.9 at any $\langle\Phi\rangle$.

In Fig. 12 we further examine the origin of the strong fluctuations in this case. It displays the snapshots of the extension vector defined by (2.34), $\left\langle\sigma_{x y}\right\rangle,\left\langle N_{1}\right\rangle$, and the following rotationally invariant shear gradient [63],

$$
S=\left[2 \sum_{i j}\left(\nabla_{i} v_{j}\right)^{2}-\left(\nabla_{x} v_{y}-\nabla_{y} v_{x}\right)^{2}\right]^{1 / 2} .
$$

Notice $S=0$ for pure rotation. At $t=500$, the angle $\theta$ exceeds $\pi / 4$ in most of the spatial points of the polymer-rich regions, resulting in $\langle\cos 2 \theta\rangle=-0.21$ and $\left\langle N_{1}\right\rangle=-0.44$. At $t=1500$, the points with $\theta<\pi / 4$ constitute a majority in the polymer-rich regions, leading to $\left\langle N_{1}\right\rangle=0.24$. These snapshots and those of the stress components clearly demonstrate the presence of stress lines forming networks, which are supported by the percolated polymer-rich regions and where the extension and the stress take large values. The typical values of $w_{1}-w_{2}$ on these lines are 0.16 at $t=500$ and 0.11 at $t=1500$. For this shear rate we can see that $\left\langle N_{1}\right\rangle$ becomes negative when the stress lines collectively rotate and the angle $\theta$ exceeds $\pi / 4$ on these lines, while for $\dot{\gamma}=0.05$ the stress lines are broken at much faster rates and $\left\langle N_{1}\right\rangle$ remains positive. On the other hand, $S$ in Fig.12 takes large values in the solvent-rich regions, indicating that the polymer-rich domains are largely rotated rather than being anisotropically deformed in shear. This tendency becomes conspicuous with decreasing shear. See the black regions of $S$ in Fig.12, where $\Phi$ is small and slipping is taking place.

Fig.13 shows a bird view of the velocity gradient $\partial v_{x} / \partial y$ (right) and the corresponding snapshot of $\Phi$ at $t=2000$, where we choose $\dot{\gamma}=0.025$, an intermediate shear rate in Fig.4. The other parameters are the same as in Figs.4-12. We recognize that the solvent-rich regions support this velocity gradient. This means that strongly deformed solvent-rich domains act as a lubricant serving to diminish the macroscopic stress or viscosity. This lubricant effect can be effective even for a small volume fraction of the solvent-rich domains because of the strong contrast of the viscoelastic properties in the two phases.
In fact Wolf and Sezen [64] presented this view to interpret their finding of a viscosity decrease which signals onset of phase separation in shear in semidilute solutions.

\section{E. Domain morphology and rheology for various $\langle\Phi\rangle$}

Next we show that the domain morphology also strongly depends on the average polymer volume fraction $\langle\Phi\rangle$. Fig.14 displays snapshots of $\Phi(\mathbf{r}, t)$ at $\dot{\gamma}=0.05$, $u=3$, and $g=1$ for three compositions, $\langle\Phi\rangle=1.2,1.7$, 2.0 , and 2.5 (from left). The time is $t=1500$, where steady states are almost reached in all these cases. The profiles of $\Phi$ in the $x$ direction at $y=128$ are also shown in the upper part. We can see that $\Phi \cong \Phi_{\mathrm{ex}} \cong 3.7$ within the polymer-rich regions. With increasing $\langle\Phi\rangle$, the collision frequency among the domains increases and the domain size decreases. In fact, $R_{\mathrm{D}}$ determined from the perimeter length is $13.75,10.83$, and 6.67 for $\langle\Phi\rangle=1.7$, 2.0 , and 2.5, respectively. Even for $\langle\Phi\rangle=1.2$ the polymer-rich domains collide frequently and the domains shapes largely deviate from sphericity. In Fig.15 we plot time evolutions of the average shear stress $\left\langle\sigma_{x y}\right\rangle$ for these values of $\langle\Phi\rangle$. In the dynamical steady state, the normalized shear viscosity increase $\Delta \eta / \eta_{0}=\eta / \eta_{0}-1$ is given by $2,4,6.5$ and 15 for $\langle\Phi\rangle=1.2,1.7,2.0$ and 2.5 , respectively. It is remarkable that the stress overshoot is nonexistent at small $\langle\Phi\rangle$ and gradually develops with increasing $\langle\Phi\rangle$.

\section{F. Deformation of sponge-like domains at small shear}

Finally we examine how the sponge-like domain structure observed by Tanaka and coworkers [6, 7] is deformed by shear flow. On the left of Fig.16 we show one example of such a domain structure without shear for $\langle\Phi\rangle=1.2$, $u=3$, and $g=1$, where the polymer-rich regions are percolated despite relatively small $\langle\Phi\rangle[\underline{9}, 10]$. The right part is the result under shear $\dot{\gamma}=0.005$ with the other parameters being common, where the applied strain $\dot{\gamma} t$ is $1,2,5$, and 10 at $t=200,400,1000$, and 2000 for the given snapshots, respectively. For this weak shear rate, the initial stage of phase separation is not much different from the case without shear but the coarsening is quickened as in Fig.4 for $\langle\Phi\rangle=2$. Here the polymer-rich domains are gradually thickened but remain highly extended in the flow direction, while the domains in Fig.14(a) are torn into pieces because of larger shear $\dot{\gamma}=0.05$. Also in this case the average shear stress $\left\langle\sigma_{x y}\right\rangle(t)$ and normal stress difference $\left\langle N_{1}(t)\right\rangle$ exhibit chaotic behavior and the product $\left\langle\sigma_{x y}\right\rangle(t) R(t)$ tends to a constant $(\sim 2.4)$. Again as in Fig.11, the negativity of the normal stress difference is conspicuous in the early stage (not shown here). 


\section{SUMMARY AND CONCLUDING REMARKS}

Though performed in two dimensions, we have numerically solved the two-fluid dynamic model of sheared semidilute polymer solutions with theta solvent, where the chain deformations are represented by the conformation tensor $W_{i j}$. The free energy density depends on $W_{i j}$ as well as the polymer volume fraction $\phi$ as in (2.3). In our simulations, the initial value of the composition is random, but the thermal noise terms in the dynamic equations are neglected for $t>0$. We summarize our main results.

(i) With varying the parameter $g$ representing the magnitude of the shear modus, we have examined spinodal decomposition as in Figs.2 and 3, which show the crossover of the domain growth from Newtonian to viscoelastic fluids. The domain growth is nearly stopped for $g \gtrsim 0.1$ at relatively large shear within our simulation time.

(ii) The domain growth in spinodal decomposition has been examined at $g=1$ with varying $\dot{\gamma}$ in Fig.4. The domain size $R_{\mathrm{D}}$ in steady states becomes finer with increasing $\dot{\gamma}$ and, as demonstrated in Fig.5, it satisfies the relation (3.2). Its theoretical derivation is not given in this paper. In Fig.6 the network, gradient, and viscous stress contributions are compared, which indicates that the polymer-rich regions mostly support the applied stress. In Fig.9 the variance $\sqrt{\left\langle\delta \Phi^{2}\right\rangle}(t)$ continues to increase even after the saturation of the stress and the domain size, which arises from slow desorption of solvent from the polymer-rich regions $[6,7]$.

(iii) Fig.7 displays the composition patterns at relatively large shear $\dot{\gamma}=0.05$. Its structure factor in Fig.8 suggests that the domains are more correlated along the shear-gradient direction rather than along the flow direction. This is consistent with the experiments [18, 21, 29, 30].

(iv) At $\dot{\gamma}=0.05, \Phi$ fluctuates in the region $|\Phi-\langle\Phi\rangle|$ between 0 and $\Phi_{\text {ex }}$ in Fig.7, exhibiting the spike-like behavior. Another notable difference is that the variance saturates rapidly above the coexistence curve 13, 36 but slowly at a larger value in Fig.9.

(v) At much smaller shear $\dot{\gamma}=0.005$, the domain size becomes larger as in Fig.10 and the stress fluctuations look much enlarged as in Fig.11. as compared to the stress curves in Fig.9 for $\dot{\gamma}=0.05$. For this small shear, percolated stress lines are formed in the polymer-rich regions as in Fig.12. There, we also notice that the non-rotational velocity gradient $S$ defined by (3.3) becomes large in the solvent-rich regions. Fig.13 shows that the velocity gradient $\partial v_{x} / \partial y$ takes large values in the solvent-rich regions, suggesting that they act as a lubricant. (vi) Fig.14 illustrates how the domain morphology depends on the average polymer volume fraction $\langle\Phi\rangle$. Fig.15 shows that the stress-strain curves also strongly depends on $\langle\Phi\rangle$.

(vii) Fig.16 shows time evolution of the sponge-like patterns without shear [6, 7] and under weak shear.

Furthermore, we make comments on experimental aspects.

(i) We have calculated the space averages of the stress components. In usual rheology experiments, however, the stress acting on the boundary surface is measured. In a dynamical steady state, the time averages of the space-averaged stress and the surface stress do coincide, but their time-dependent fluctuations can be different. We note that the time-dependent fluctuations in these quantities become significant when the fluctuating entities are of very large sizes. Here it is worth noting that more than three decades ago Lodge [26] reported abnormal temporal fluctuations of the normal stress difference at a hole of $1 \mathrm{~mm}$ diameter from a polymer solution contained in a cone-plate apparatus. He ascribed its origin to growth of inhomogeneities or gel-like particles.

(ii) Below the coexistence curve, we have shown sharp stress overshoots at relatively strong shear in Fig.9. However, the stress-strain curves at relatively small shear in Fig.11 and those corresponding to Fig.16 (not shown) are more complex, where the frequent negativity of the normal stress is conspicuous. In the snapshots in Figs. 7, 10, and 16 the domains are collectively rotated as a whole in the early stage. Thus the first normal stress might become negative (at least) after its first peak.

(iii) Disappearance of the stress peaks at small average volume fractions in Fig.15 should also be observed.

(iv) Experiments are needed on establishment of dynamical steady states with the characteristic heterogeneity length given by (3.2). Strong deviations of the polymer-rich domains from sphericity are also characteristic. These results gained from the simulations could be compared with microscope observations [17, 29].

In future, we should perform simulations to examine heterogeneous fluctuations in various sheared complex fluids such as polymer blends or wormlike micellar systems.

\section{Acknowledgments}

We would like to thank Prof. Takeji Hashimoto and Prof. Hajime Tanaka for valuable discussions. This work is supported by Grants in Aid for Scientific Research and for the 21st Century COE project (Center for Diversity and Universality in Physics) from the Ministry of Education, Culture, Sports, Science and Technology of Japan.
[1] L.A. Utracki, Polymer Alloys and Blends. Thermodynamics and Rheology (Hanser Publishers, Munich, 1990).
[2] A. Onuki, Phase Transition Dynamics, (Cambridge Uni- 
versity Press, Cambridge, 2002).

[3] G. Müller, D. Schwahn, H. Eckerlebe, J. Rieger, and T. Springer, ibid. 104, 5826 (1996).

[4] N. Toyoda, M Takenaka, S. Saito, and T. Hashimoto, Polymer 42, 9193 (2001); M Takenaka, H. Takeno, H. Hasegawa, S. Saito, T. Hashimoto, and M. Nagao, Phys. Rev. E 65, 021806 (2002).

[5] M. Doi and A. Onuki, J. Physique II 2, 1631 (1992).

[6] H. Tanaka, Phys. Rev. Lett. 71, 3158 (1993); J. Chem. Phys. 100, 5253 (1994).

[7] H. Tanaka, Phys. Rev. Lett. 76, 787 (1996).

[8] A. Onuki and T. Taniguchi, J. Chem. Phys. 106, 5761 (1997).

[9] T. Taniguchi and A. Onuki, Phys. Rev. Lett. 77, 4910 (1996).

[10] H. Tanaka and T. Araki, Phys. Rev. Lett. 78, 4966 (1997); Macromolecules 34, 1953 (2001).

[11] J. Zhang, Z. Zhang, Hongdong Zhang, and Yuliang Yang, Phys. Rev. E 64, 051510 (2001).

[12] R.G. Larson, Rheol. Acta. 31, 497 (1992).

[13] A. Onuki, J. Phys. C 9, 6119 (1997).

[14] A. Onuki and K. Kawasaki, Ann. Phys. (NY) 121, 456 (1979).

[15] D. Beysens and M. Gbadamassi, Phys. Rev. A 22, 2250 (1980).

[16] K. Asakawa and T. Hashimoto, J. Chem. Phys. 105, 5216 (1996).

[17] T. Hashimoto, K. Matsuzaka, E. Moses, and A. Onuki, Phys. Rev. Lett. 74, 126 (1995).

[18] X.L. Wu, D.J. Pine, and P.K. Dixon, Phys. Rev. Lett. 68, 2408 (1991).

[19] T. Hashimoto and K. Fujioka, J. Phys. Soc. Jpn. 60, 356 (1991); T. Hashimoto and T. Kume, ibid. 61, 1839 (1992); H. Murase, T. Kume, T. Hashimoto, Y. Ohta, and T. Mizukami, Macromolecules 28, 7724 (1995).

[20] T. Kume, T. Hattori, and T. Hashimoto, Macromolecules 30, 427 (1997).

[21] S. Saito and T. Hashimoto, J. Chem. Phys. 114, 10531 (2001); S. Saito, T. Hashimoto, I. Morfin, P. Linder, and F. Boue, ibid. 35, 445 (2002).

[22] J. van Egmond, D.E. Werner, and G.G. Fuller, J. Chem. Phys. 96, 7742 (1992); J. van Egmond and G. Fuller, Macromolecules 26, 7182 (1993).

[23] A.I. Nakatani, J.F. Douglas, Y.-B. Ban, and C.C. Han, J. Chem. Phys. 100, 3224 (1994).

[24] I. Morfin, P. Linder, and F. Boue, Macromolecules 32, 7208 (1999).

[25] K. Migler, C. Liu, and D.J. Pine, Macromolecules 29, 1422 (1996).

[26] A.S. Lodge, Polymer 2, 195 (1961).

[27] A. Peterlin and D.T. Turner, J. Polym. Sci., Polym. Lett. 3, 517 (1965).

[28] J.J. Magda, C.-S. Lee, S.J. Muller, and R.G. Larson, Macromolecules 26, 1696 (1993).

[29] E. Moses, T. Kume, and T. Hashimoto, Phys. Rev. Lett. 72, 2037 (1994).

[30] E.K. Hobbie, H.S. Jeon, H. Wang, H. Kim, D.J. Stout and C.C. Han, J. Chem. Phys. 117, 6350 (2002).

[31] E. Helfand and G.H. Fredrickson, Phys. Rev. Lett. 62, 2468 (1989).

[32] A. Onuki, Phys. Rev. Lett. 62, 2472 (1989); A. Onuki, J. Phys. Soc. Jpn. 59, 3423 (1990); 59, 3427 (1990).

[33] S.T. Milner, Phys. Rev. Lett. 66, 1477 (1991); Phys. Rev. E 48, 3674 (1993).
[34] H. Ji and E. Helfand, Macromolecules 28, 3869 (1995).

[35] G.H. Fredrickson, J. Chem. Phys. 117, 6810 (2002).

[36] A. Onuki, R. Yamamoto, and T. Taniguchi, J. Physique II 7, 295 (1997); Progress in Colloid \& Polymer Science 106, 150 (1997).

[37] T. Okuzono, Modern Phys. Lett. 11, 379 (1997).

[38] X. -F. Yuan and L. Jupp, Europhys. Lett. 60, 691 (2002).

[39] L. Jupp, T. Kawakatsu, and X.-F. Yuan, J. Chem. Phys. 119, 6361(2003).

[40] S. M. Fielding and P. D. Olmsted, Phys. Rev. E 68, 036313 (2003).

[41] B. Chakrabarti, M. Das, C. Dasgupta, S. Ramaswamy, and A.K. Sood, Phys. Rev. Lett. 92, 055501 (2004).

[42] F. Pignon, A. Magnin, and J.-M. Piau, J. Rheol. 40, 573; (1996); R. Bandyopadhyay, and A.K. Sood, Europhys. Lett. 56, 447 (2001); J.-B. Salmon, S. Manneville, and A. Colin, Phys. Rev. E 68051504 (2003).

[43] M.E. Cates, D.A. Head, and A. Ajdari, Phys. Rev. E 66, 025202 (2002); G. Picard, A. Ajdari, L. Bocquet, and F. Lequeux: Phys. Rev. E 66, 051501 (2002).

[44] X.-F. Yuan, Europhys. Lett., 46, 542 (1999); P. D. Olmsted, O. Radulescu, and C.-Y.D. Lu, J. Rheol. 44, 257 (2000).

[45] P.G. de Gennes, Scaling Concepts in Polymer Physics (Ithaca, Cornell University Press, 1980).

[46] T. Ohta, H. Nozaki and M. Doi, J. Chem. Phys. 93, 2664 (1990); D.H. Rothman, Europhys. Lett. 14, 337 (1991); P. Padilla and S. Toxvaerd, J. Chem. Phys. 106, 2342 (1997); A. J. Wagner and J. M. Yeomans, Phys. Rev. E 59, 4366 (1999).

[47] L. Berthier, Phys. Rev. E 63, 051503 (2001).

[48] A. Frischknecht, Phys. Rev. E 56, 6970 (1997); Phys. Rev. E 58, 3495 (1998).

[49] K.B. Migler, Phys. Rev. Lett. 86, 1023 (2001).

[50] M. Grmela, Phys. Lett. A 130, 81 (1988); H. C. Öttinger and M. Grmela Phys. Rev. E 56, 6633 (1997).

[51] A.N. Beris and B.J. Edwards, Thermodynamics of Flowing Systems (Oxford: Oxford University Press, 1994).

[52] M. Adam and M. Delsanti, J. Physique 44, 1185 (1983); ibid.45, 1513 (1984).

[53] Y. Takahashi, Y. Isono, I. Noda, and M. Nagasawa, Macromolecules 18, 1002 (1985).

[54] R.G. Larson, The Structure and Rheology of Complex Fluids (Oxford, 1999).

[55] The factor $1 /(1+Q)$ in (2.21) serves to stabilize numerical integration of (2.11).

[56] J.L. White and A.B. Metzner, J. Appl. Polym. Sci., 8, 1367 (1963).

[57] A. Onuki, Phys. Rev. A 35, 5149 (1987).

[58] We have also performed simulations with the mesh size taken to be $\Delta x=\ell / \sqrt{2}$ and $\ell / 2$. Then the curves in the bottom plate in Fig. 7 coincide within $5 \%$ without any qualitative differences. The behavior of $\Phi$ in the spikelike regions in Fig.7 are well described even with $\Delta x=\ell$.

[59] A. Onuki, J. Phys. Soc. Jpn. 66, 1836 (1997).

[60] At $t=0$ we consider the variance of the deviation $\int d \boldsymbol{r} \delta \phi / \phi_{\mathrm{c}} \ell^{d}$ averaged in one unit cell. Its thermal value is of order $\Phi^{-1 / 2}$ in the semidilue theta condition.

[61] T. Baumberger, F. Perrot, and D. Beysens, Phys. Rev. A 46, 7636 (1992).

[62] A. Onuki, Europhys. Lett. 28, 175 (1994). In the Newtonian case, the ratio $C_{\mathrm{New}} / \gamma$ is of order $\phi$ if the droplet volume fraction is considerably smaller than $1 / 2$ and if 
the viscosity ratio $\eta_{1} / \eta_{2}$ is close to 1 . If $\eta_{1} / \eta_{2}$ much deviates from $1, C_{\mathrm{New}}$ is a complicated function of $\phi$ and $\eta_{1} / \eta_{2}$. For $\phi \sim 1 / 2$ the domains can be extended into very long cylinders resulting in a string phase [17, 48, 49].

[63] Under (2.10), $S$ in (3.3) and $\nabla_{x} v_{y}-\nabla_{y} v_{x}$ are the strain rates invariant with respect to rotation of the reference frame in two dimensions.

[64] B.A. Wolf and M.C. Sezen, Macromolecules 10, 1010 (1977); B.A. Wolf and R. Jend, ibid. 12, 732 (1979). 


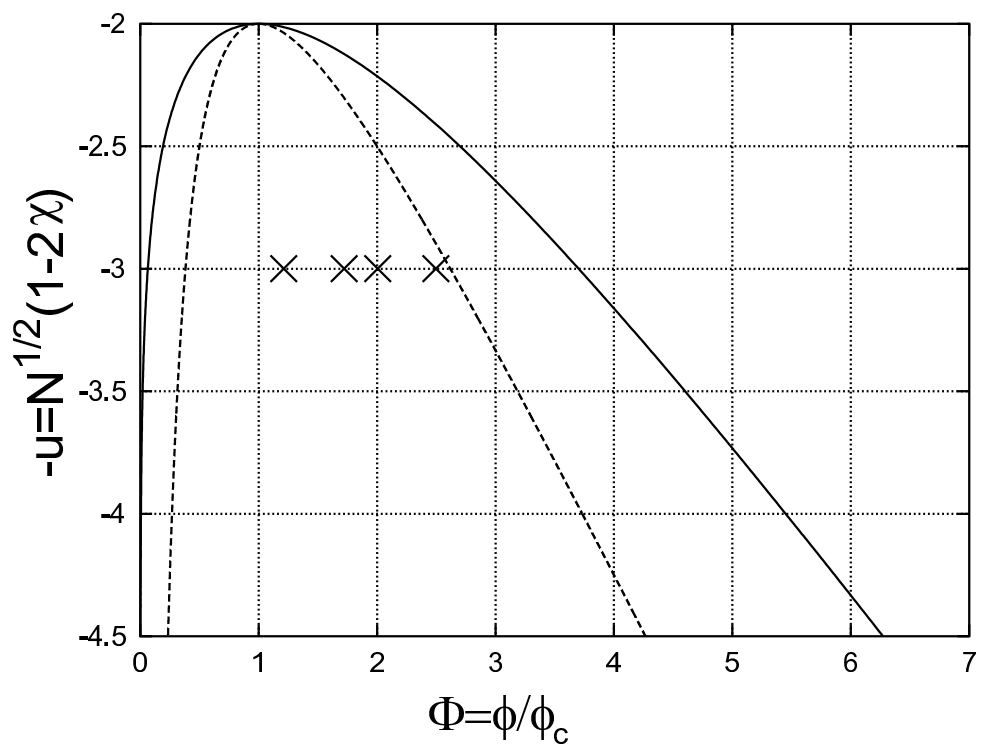

FIG. 1: Coexistence curve (solid line) and spinodal curve (dashed line) for polymer solutions obtained from 2.1) in the plane of $-u=N^{1 / 2}(1-2 \chi)$ and $\Phi=\phi / \phi_{c}$. The points $(\times)$ represent the initial states of our simulations.

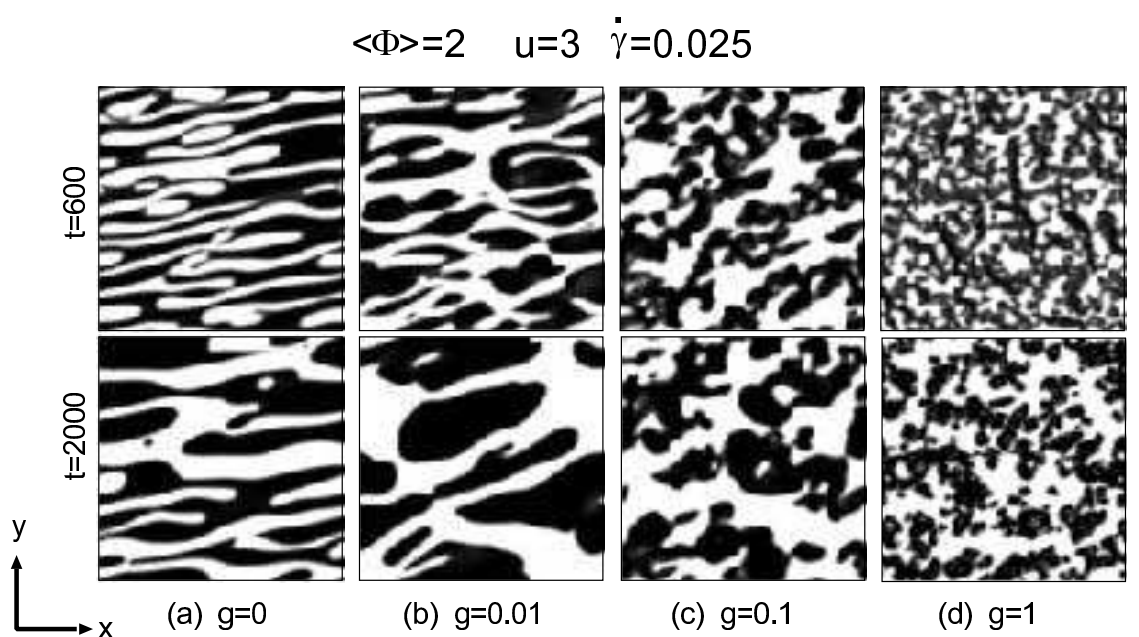

FIG. 2: Crossover of domain patterns of $\Phi(\mathbf{r}, t)$ below the spinodal curve with increasing $g$ from 0 to 1 for $u=3$ and $\langle\Phi\rangle=2$ in shear flow with $\dot{\gamma}=0.025$. 


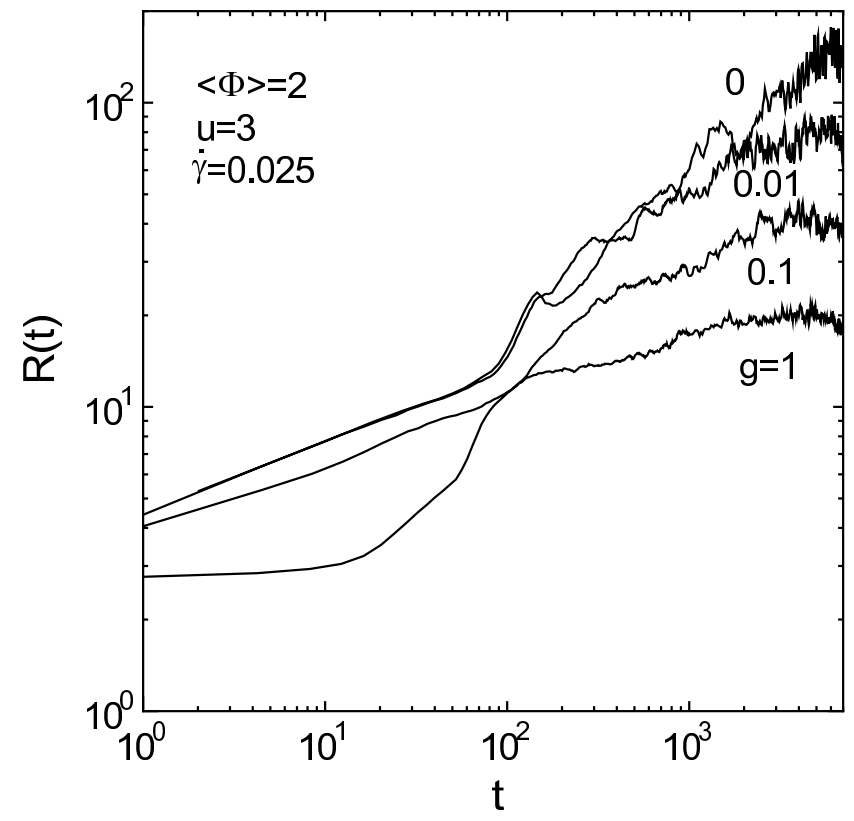

FIG. 3: Time evolution of the domain size $R(t)$ (= the inverse of the perimeter length density) below the spinodal curve with increasing the shear modulus as $g=0,0.01,0.1$, and 1 in shear flow with $\dot{\gamma}=0.025$. The other parameters are the same as those in Fig.2. The domain growth is nearly stopped for $g=1$ and 0.1 within the simulation time.

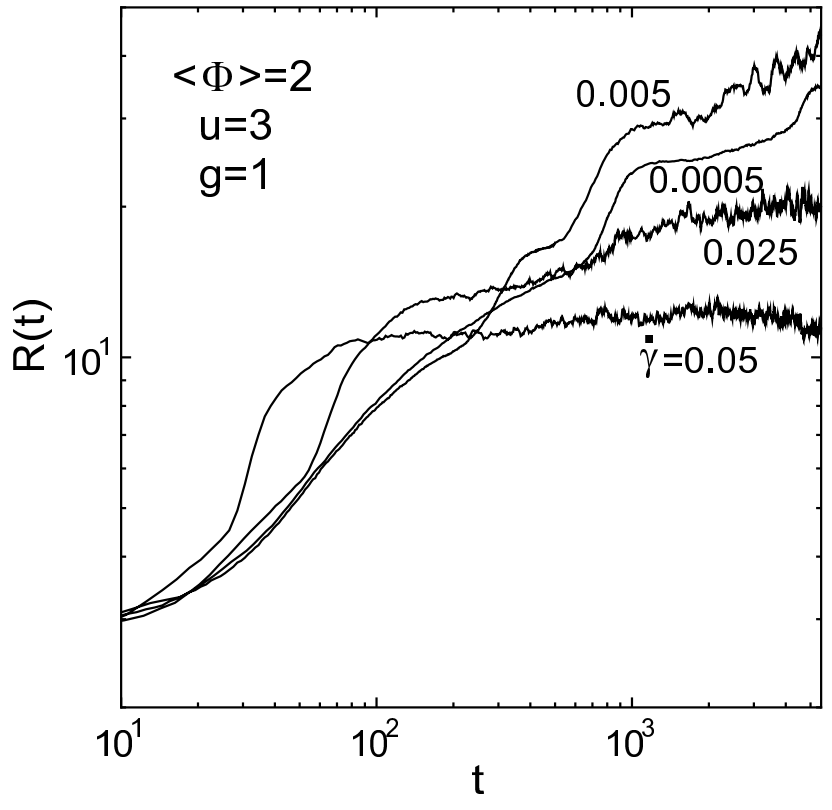

FIG. 4: Time evolution of the domain size $R(t)$ below the spinodal curve for $g=1, u=3$, and $\langle\Phi\rangle=2$. Here $\dot{\gamma}=0.0005$, $0.005,0.025$, and 0.05 . At small shear rates flow-induced coagulation accelerates the domain growth as demonstrated by the curve of $\dot{\gamma}=0.005$. For $\dot{\gamma} \gg 0.005$, shear-induced domain breakup becomes dominant and dynamical steady states are realized at smaller domain sizes. 


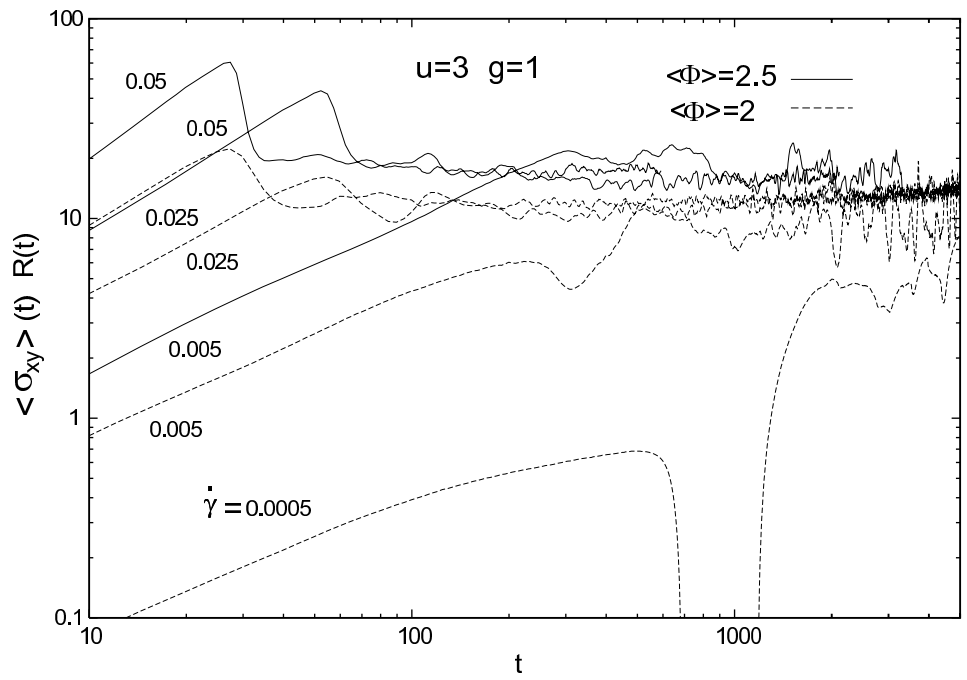

FIG. 5: Time evolution of $\left\langle\sigma_{x y}\right\rangle(t) R(t)$ below the spinodal curve at various shear rates for $g=1$ and $u=3$. Here $\langle\Phi\rangle=2$ (solid lines) or $\langle\Phi\rangle=2.5$ (dotted lines). At long times the curves tend to fluctuate around $15-20$ confirming (3.4).

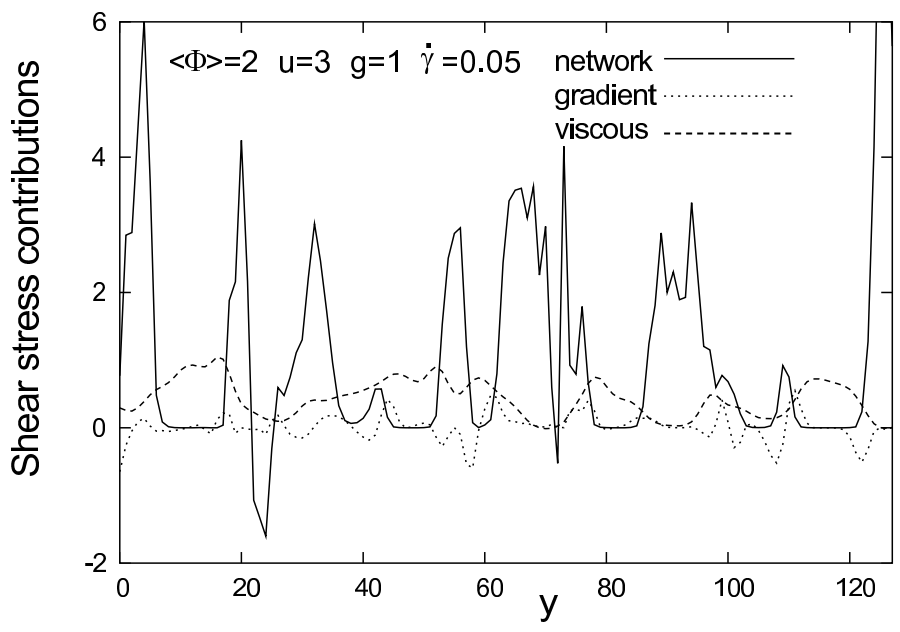

FIG. 6: Three contributions, $\sigma_{\mathrm{p} x y}$ (network), $-4\left(\nabla_{x} \Phi\right)\left(\nabla_{y} \Phi\right) / \Phi$ (gradient), and $4\left(\nabla_{x} v_{y}+\nabla_{y} v_{x}\right)$ (viscous) to the local shear stress $\sigma_{x y}(x, y)$ in $(2.40)$ in the range $0<y<128$ at $x=0$ for $u=3,\langle\Phi\rangle=2$, and $\dot{\gamma}=0.05$. The network stress is overwhelming in the polymer-rich regions, while the viscous one is relatively large in the solvent-rich regions. The gradient contribution is appreciable only in the interface regions, where the three contributions are of the same order. 


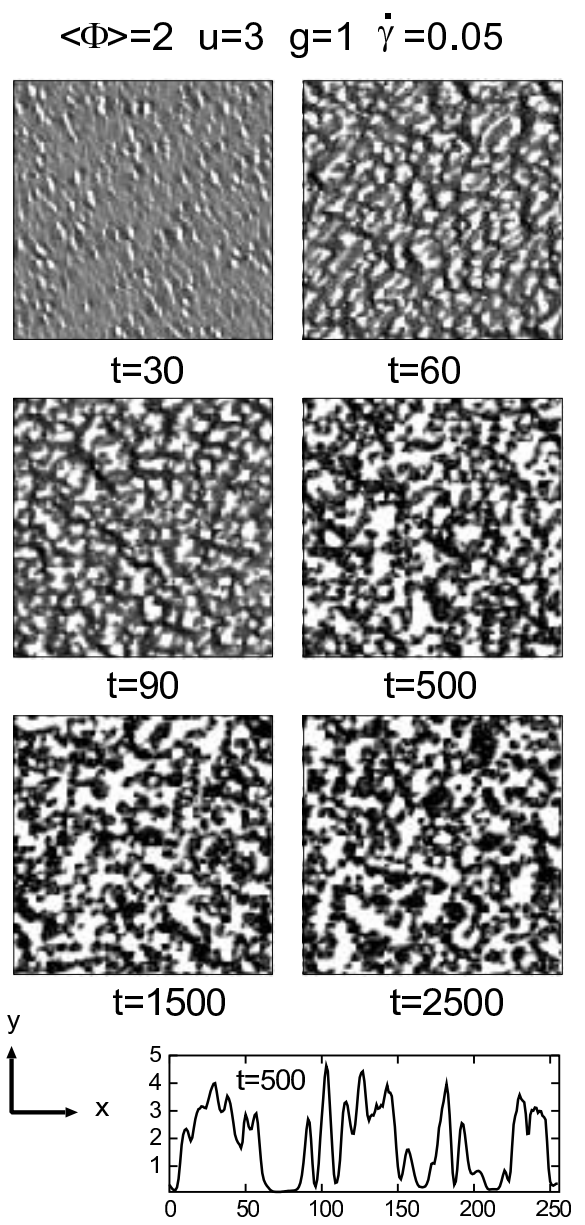

FIG. 7: Time evolution of $\Phi(\mathbf{r}, t)$ at $\dot{\gamma}=0.05$ for $u=3,\langle\Phi\rangle=2$, and $g=1$ below the spinodal curve. The bottom figure is the profile at $t=500$ in the $x$ direction at $y=128$. The domain size evolution is given by the curve of the largest shear $\dot{\gamma}=0.05$ in Fig.4.
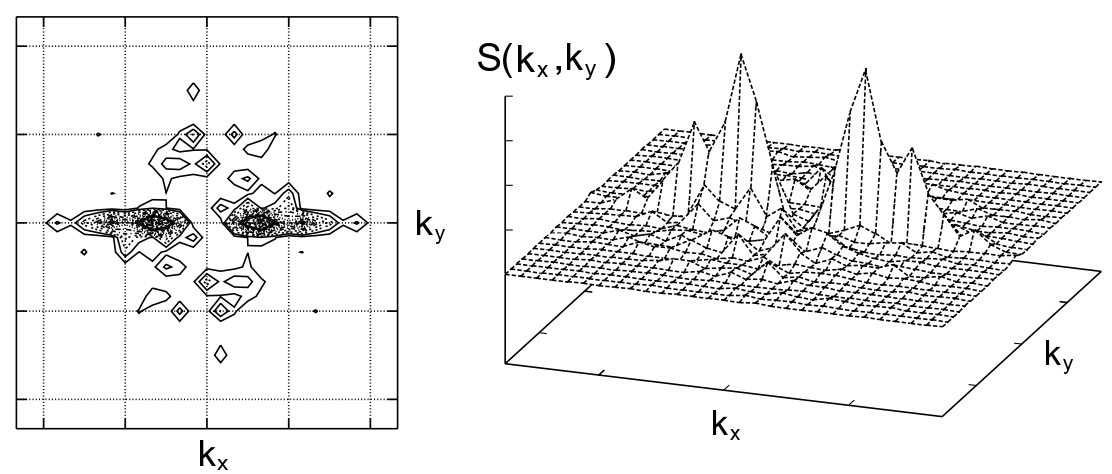

FIG. 8: Structure factor $S(\boldsymbol{k})$ for the composition patterns in Fig.7 in the dynamical steady state. 


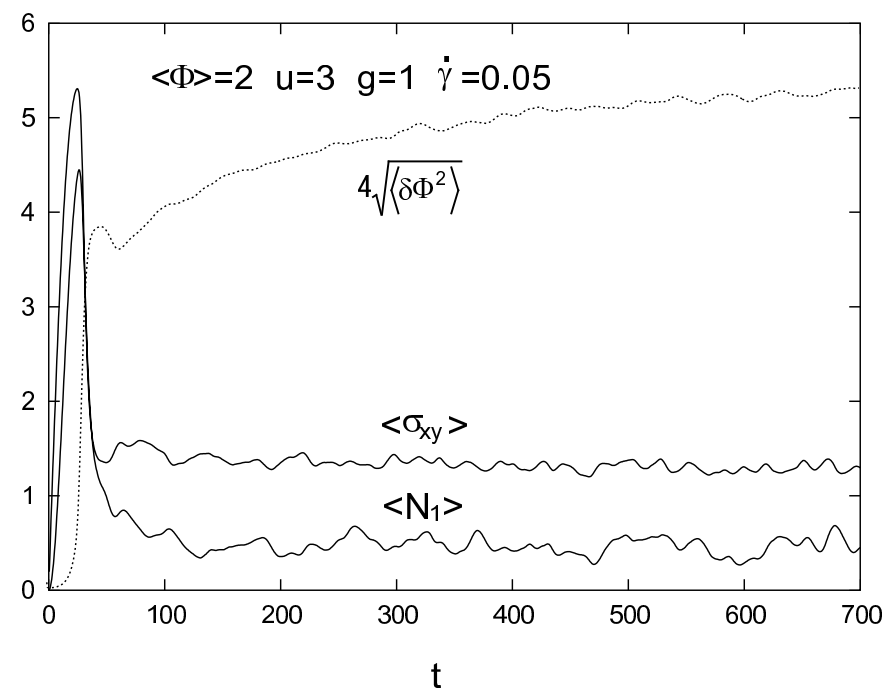

FIG. 9: Time evolution of the average shear stress $\left\langle\sigma_{x y}\right\rangle(t)$, the average normal stress difference $\left\langle N_{1}\right\rangle(t)$, and the average variance $\sqrt{\left\langle\delta \Phi^{2}\right\rangle}(t)$ (dotted line) at $\dot{\gamma}=0.05$ for $u=3,\langle\Phi\rangle=2$, and $g=1$ below the spinodal curve.

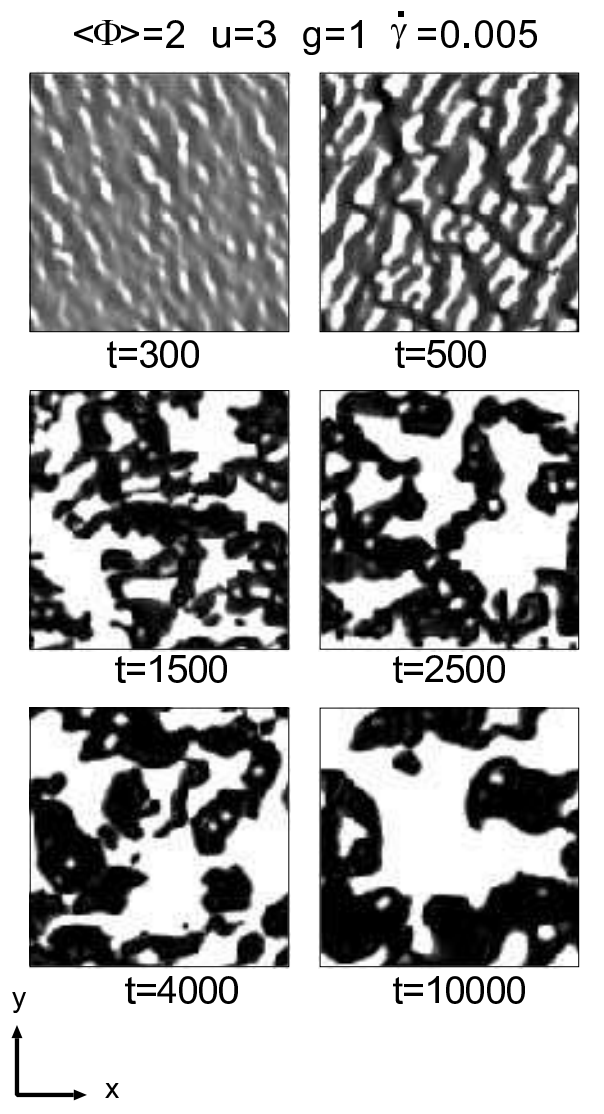

FIG. 10: Time evolution of $\Phi(\mathbf{r}, t)$ at $\dot{\gamma}=0.005$ for $u=3,\langle\Phi\rangle=2$, and $g=1$ below the spinodal curve. For this shear rate Fig. 4 shows that the domain size increases up to the system size at $t=10^{4}$. 


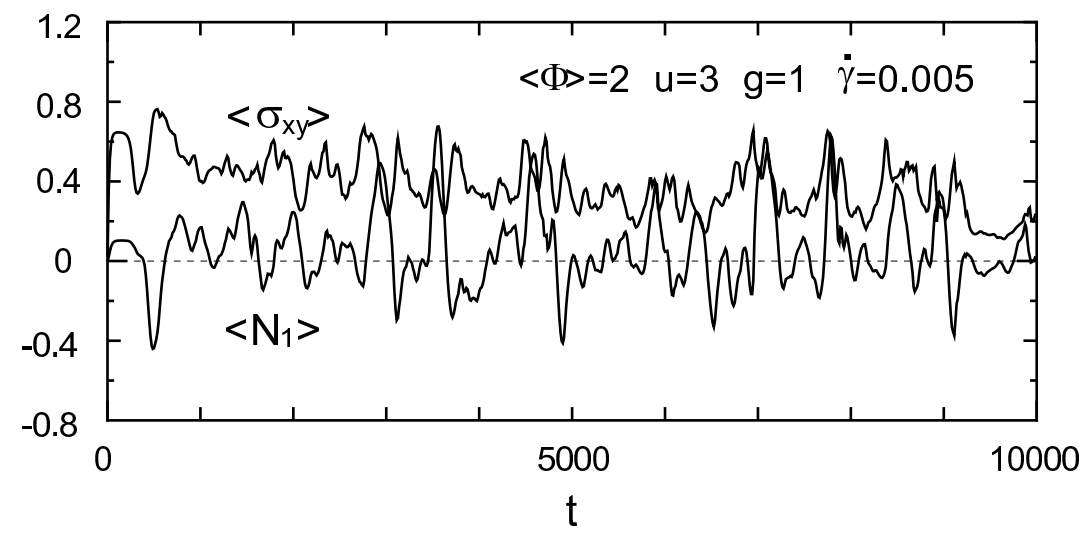

FIG. 11: Chaotic time evolution of the average shear stress $\left\langle\sigma_{x y}\right\rangle(t)$ and the average normal stress difference $\left\langle N_{1}\right\rangle(t)$ at $\dot{\gamma}=0.005$ in the run which produced Fig.10. For this weak shear the deviatoric stress components exhibit large fluctuations and $\left\langle N_{1}\right\rangle(t)$ frequently takes negative values.

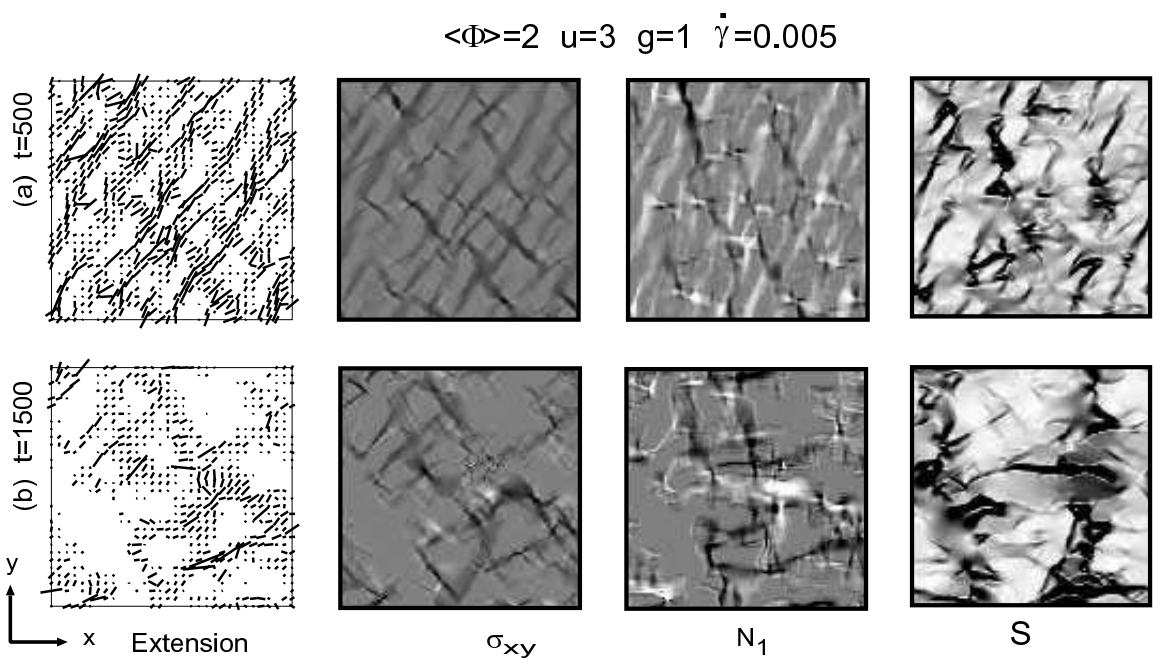

FIG. 12: Patterns of the extension, the shear stress, the normal stress difference, and the rotationally invariant shear gradient (3.3) at $t=500$ (top) and $t=1500$ (bottom). The corresponding composition patterns are shown in Fig.10. We can see stress lines with large values of the extension and the deviatoric stress components. The $S$ is large in solvent-rich slipping regions.

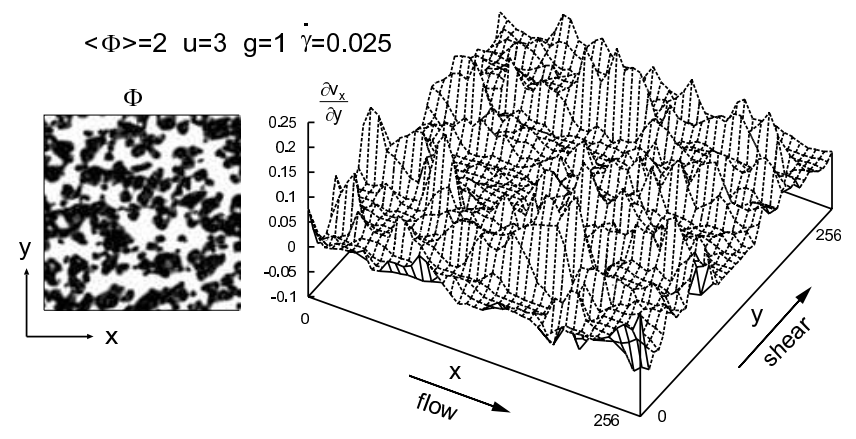

FIG. 13: $\Phi(\mathbf{r}, t)$ (left) and $\partial v_{x}(\mathbf{r}, t) / \partial y$ (right) at $\dot{\gamma}=0.025$ for $u=3,\langle\Phi\rangle=2$, and $g=1$ in a steady state. This velocity gradient takes large values where slipping is taking place in the percolated solvent-rich region. 


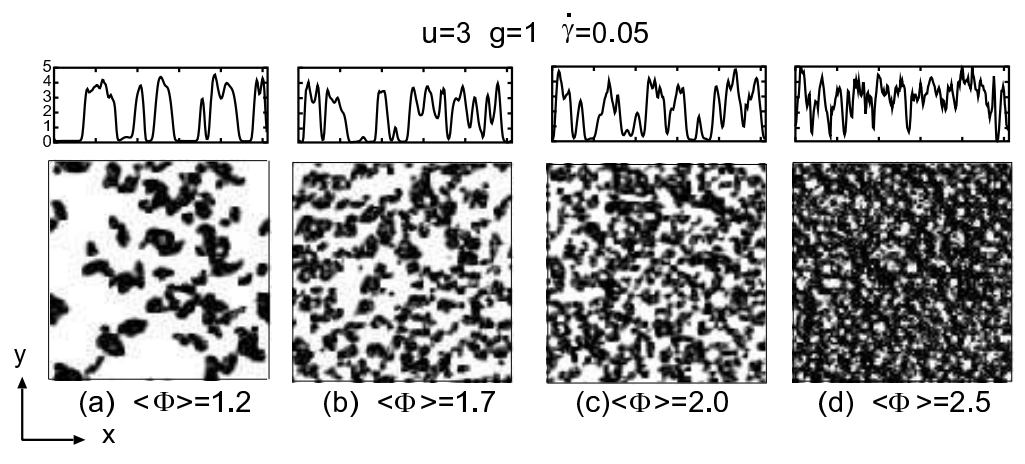

FIG. 14: Snapshots of $\Phi(\mathbf{r}, t)$ for $\langle\Phi\rangle=1.2,1.7,2.0$, and 2.5 in steady states at $\dot{\gamma}=0.05$. The profiles of $\Phi$ in the $x$ direction at $y=128$ are also shown.

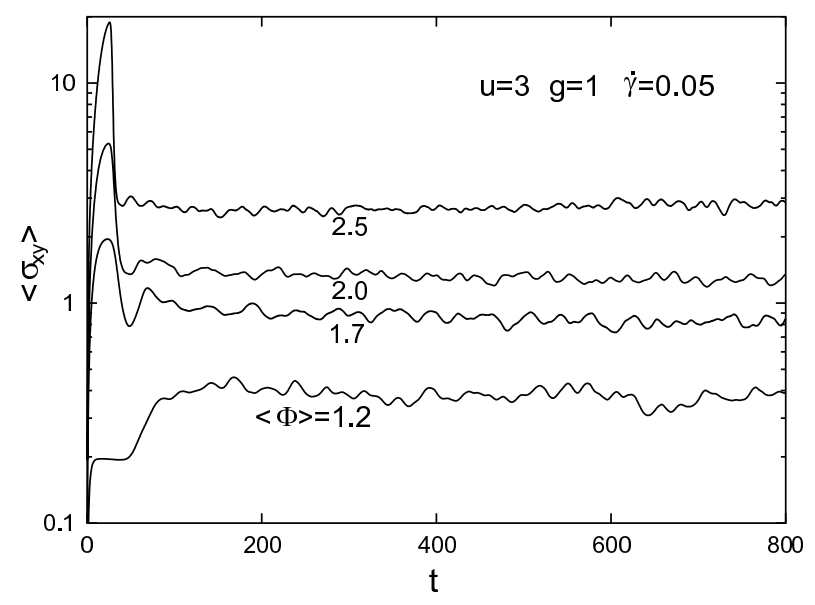

FIG. 15: Time evolution of $\left\langle\sigma_{x y}\right\rangle(t)$ at various $\langle\Phi\rangle$. The corresponding composition patters are shown in Fig.14. The overshoot disappears at small $\langle\Phi\rangle$.

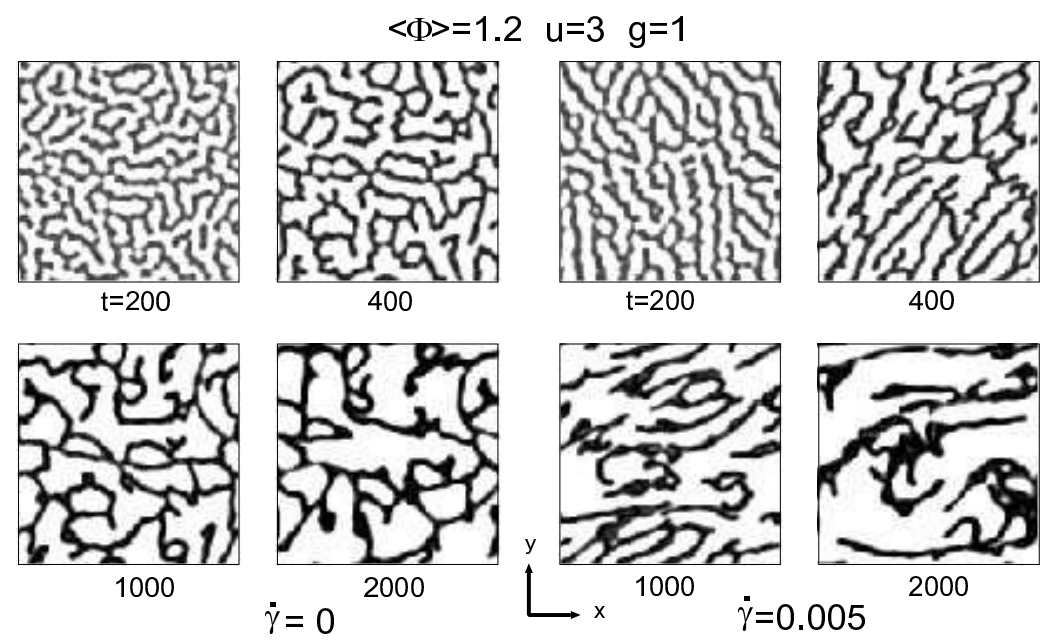

FIG. 16: Sponge-like domain structures without shear (left) and under shear $\dot{\gamma}=0.005$ (right) for $\langle\Phi\rangle=1.2, u=3$, and $g=1$. 